\section{Korrespondenzadresse}

\section{PD Dr. J. Hübner}

Arbeitsgruppe Integrative Onkologie

Dr. Senckenbergisches Chronomedizinisches Institut

J.W. Goethe-Universität

Theodor-Stern-Kai 7

60596 Frankfurt

Huebner@med.uni-frankfurt.de

\section{Einhaltung ethischer Richtlinien}

Interessenkonflikt. J. Hübner gibt an, dass kein Interessenkonflikt besteht.

Der Beitrag enthält keine Studien an Menschen oder Tieren.

\section{Literatur}

1. http://www.lilly-pharma.de/download.php?file=fileadmin/media/lilly/fachinfo/gemzar\&code $=29881$ bc 465 bdab57bcf9f20bbfbfa227. Zugegriffen: 22. Feb. 2015

2. Tröger W, Jezdić S, Ždrale Z et al (2009) Quality of life and neutropenia in patients with early stage; breast cancer: a randomized pilot study comparing additional treatment with mistletoe extract to chemotherapy alone. Breast Cancer 3:35-45

3. Tröger W, Ždrale Z, Stanković N, Matijašević M (2012) Five-year follow-up of patients with early stage breast cancer after a randomized study comparing additional treatment with viscum album (L.) extract to chemotherapy alone. Breast Cancer 6:173-180

4. Tröger W, Galun D, Reif M et al (2013) Viscum album [L.] extract therapy in patients with locally advanced or metastatic pancreatic cancer: a randomised clinical trial on overall survival. Eur J Cancer 49:37883797

5. Tröger W, Galun D, Reif M et al (2014) Quality of life of patients with advanced pancreatic cancer during treatment with mistletoe - a randomized controlled trial. Dtsch Arztebl Int 111:493-502. doi:10.3238/ arztebl.2014.0493

6. Gabius HJ, Darro F, Remmelink M et al (2001) Evidence for stimulation of tumor proliferation in cell lines and histotypic cultures by clinically relevant low doses of the galactoside-binding mistletoe lectin, a component of proprietary extracts. Cancer Invest 19(2):114-126

7. Kelter G, Schierholz JM, Fischer IU, Fiebig H-H (2007) Anticancer Res 27:223-234

8. Kunze E, Schulz H, Adamek M, Gabius HJ (2000) Long-term administration of galactoside-specific mistletoe lectin in an animal model: no protection against N-butyl-N-(4-hydroxybutyl)-nitrosamine-induced urinary bladder carcinogenesis in rats and no induction of a relevant local cellular immune response. J Cancer Res Clin Oncol 126(3):125-138

9. Kovacs E (2004) Effects of Viscum album extract therapy in patients with cancer: relation with interleukin-6, soluble interleukin-6 receptor, and soluble gp130. J Altern Complement Med 10(2):241-246

10. Styczynski J, Wysocki M (2006) Alternative medicine remedies might stimulate viability of leukemic cells. Pediatr Blood Cancer 46(1):94-98

11. Timoshenko AV, Lan Y, Gabius HJ, Lala PK (2001) Immunotherapy of $\mathrm{C} 3 \mathrm{H} / \mathrm{HeJ}$ mammary adenocarcinoma with interleukin-2, mistletoe lectin or their combination. effects on tumour growth, capillary leakage and nitric oxide (NO) production. Eur J Cancer 37(15):1910-1920

Onkologe 2015 $\cdot 21: 344-346$

DOI 10.1007/s00761-015-2950-0

Online publiziert: 21. März 2015

○) Springer-Verlag Berlin Heidelberg 2015

\title{
R.-D. Hofheinz
}

TagesTherapieZentrum (TTZ) am Interdisziplinären Tumorzentrum Mannheim (ITM),

Universitätsmedizin Mannheim, Ruprecht-Karls-Universität Heidelberg, Mannheim

\section{Erratum zu: Perioperative (Radio-)Chemotherapie des lokal fortgeschrittenen Rektumkarzinoms}

In Tabelle 2 wurden leider die beiden Zahlenwerte zur präoperativen Radiotherapie und der präoperativen Radiochemotherapie in der Zeile Lokalrezidivrate der FFCD-9203-Studie vertauscht. Die unten stehende Tabelle gibt die korrekten Daten wieder.

Die Autoren bitten das Versehen zu entschuldigen.

\section{Korrespondenzadresse}

Prof. Dr. R.-D. Hofheinz

TagesTherapieZentrum (TTZ) am Interdisziplinären Tumorzentrum Mannheim (ITM), Universitätsmedizin Mannheim, Ruprecht-Karls-Universität Heidelberg Theodor-Kutzer Ufer 1-3, 68167 Mannheim ralf.hofheinz@umm.de

Die Online-Version des Originalartikels können Sie unter http://dx.doi.org/10.1007/s00761-0142767-2 finden.

Tab. 1 Präoperative, konventionell fraktionierte Radiotherapie mit oder ohne simultane Chemotherapie mit 5-FU/Leucovorin: Ergebnisse der EORTC-22921-Studie und der FFCD9203-Studie

\begin{tabular}{|llll}
\hline Fünfjahresergebnisse & Präoperative RT & Präoperative RChT & p-Wert \\
\hline EORTC 22921 (n=1011; [5]) & & & \\
\hline Pathologische CR & $5,3 \%$ & $13,7 \%$ & $<0,001$ \\
\hline ypN0 & $60,5 \%$ & $71,9 \%$ & $<0,001$ \\
\hline Tumorgröße (median) & $30 \mathrm{~mm}$ & $25 \mathrm{~mm}$ & $<0,0001$ \\
\hline Sphinktererhalt & $52,4 \%$ & $55,6 \%$ & 0,05 \\
\hline Lokalrezidivrate & $17 \%$ & $8 \%$ & 0,002 \\
\hline Gesamtüberleben & $64,8 \%$ & $65,6 \%$ & 0,79 \\
\hline FFCD 9203 (n=762; [16]) & & & $<0,0001$ \\
\hline Pathologische CR & $3,6 \%$ & $11,4 \%$ & 0,85 \\
\hline ypN0 & $65 \%$ & $67 \%$ & $<0,0001$ \\
\hline Grad-3/4-Akuttoxizität & $2,9 \%$ & $14,9 \%$ & 0,84 \\
\hline Sphinktererhalt & $54,4 \%$ & $52,4 \%$ & 0,004 \\
\hline Lokalrezidivrate & $16,5 \%$ & $8,1 \%$ & 0,68
\end{tabular}




\section{Hier steht eine Anzeige.}

算 Springer 